\title{
Comparative genomic analysis of clinical and environmental strains provides insight into the pathogenicity and evolution of Vibrio parahaemolyticus
}

\author{
Lei Li ${ }^{1,4}$, Hin-chung Wong ${ }^{2}$, Wenyan Nong ${ }^{1}$, Man Kit Cheung ${ }^{1}$, Patrick Tik Wan Law ${ }^{1}$, Kai Man Kam³ \\ and Hoi Shan Kwan ${ }^{1 *}$
}

\begin{abstract}
Background: Vibrio parahaemolyticus is a Gram-negative halophilic bacterium. Infections with the bacterium could become systemic and can be life-threatening to immunocompromised individuals. Genome sequences of a few clinical isolates of $V$. parahaemolyticus are currently available, but the genome dynamics across the species and virulence potential of environmental strains on a genome-scale have not been described before.

Results: Here we present genome sequences of four $V$. parahaemolyticus clinical strains from stool samples of patients and five environmental strains in Hong Kong. Phylogenomics analysis based on single nucleotide polymorphisms revealed a clear distinction between the clinical and environmental isolates. A new gene cluster belonging to the biofilm associated proteins of $V$. parahaemolyticus was found in clincial strains. In addition, a novel small genomic island frequently found among clinical isolates was reported. A few environmental strains were found harboring virulence genes and prophage elements, indicating their virulence potential. A unique biphenyl degradation pathway was also reported. A database for $V$. parahaemolyticus (http://kwanlab.bio.cuhk.edu.hk/vp) was constructed here as a platform to access and analyze genome sequences and annotations of the bacterium.

Conclusions: We have performed a comparative genomics analysis of clinical and environmental strains of $V$. parahaemolyticus. Our analyses could facilitate understanding of the phylogenetic diversity and niche adaptation of this bacterium.
\end{abstract}

Keywords: Vibrio parahaemolyticus, Comparative genomics, Clinical, Environment

\section{Background}

Foodborne diseases remain a serious public health problem worldwide. In Hong Kong, infections due to foodborne pathogens are also a common and important public health issue. Among all causative agents resulting in foodborne outbreaks, bacteria have caused more than $80 \%$ of the cases. In 2006, the Centre for Health Protection of Hong Kong revealed more than 800 local foodborne outbreaks due to bacterial causative agents, inflicting more than 3000 people [1]. Vibrio parahaemolyticus, a Gram-

\footnotetext{
*Correspondence: hoishankwan@cuhk.edu.hk

'School of Life Sciences, The Chinese University of Hong Kong, Shatin, New Territories, Hong Kong SAR, People's Republic of China

Full list of author information is available at the end of the article
}

negative pathogenic halophilic bacterium, is the most prevalent in certain Asian areas and causes food poisoning, occasionally outbreaks, especially in the hot season [2].

$V$. parahaemolyticus is well known as the causative agent of the most prevalent food poisoning in Asia since the mackerel-borne outbreak in 1959 [3]. V. parahaemolyticus infections arise from the consumption of raw or undercooked seafood, typically causing gastroenteritis [4]. Infections can become systemic and can be lifethreatening to immunocompromised individuals [5].

$V$. parahaemolyticus strains isolated from diarrheal patients produce thermostable direct hemolysin (TDH), TDH-related hemolysin (TRH), or both, while isolates from the environment rarely contain genes encoding 
these proteins. The TDH is the major virulence gene of $V$. parahaemolyticus and present in most of the clinical Kanagawa phenonemon (KP)-positive strains. In addition to its hemolytic activity, enterotoxicity, cytotoxicity as well as cardiotoxicity have been confirmed in TDH [6]. TDH-related hemolysin (TRH) is produced by KPnegative strains, encoded by trh gene, with about $67 \%$ amino acid sequence homology and immunologically related with TDH [7]. TRH is hemolytic, heat labile and is suspected to play an unclarified role in the diarrhea caused by these KP-negative strains. After the bacteria produce TDH and lipopolysaccharides released from a dead cell, human host would show strong systemic responses and produce mucosal B-cells against these antigens. However, recent study has also revealed that $V$. parahaemolyticus could profoundly disturb epithelial barrier function in Caco-2 cells with other virulence factors, in the absence of TDH [8]. $V$. parahaemolyticus also encodes two type III secretion systems (T3SS), which are located in different chromosomes. The contribution of these two T3SS has previously been characterized in animal models $[9,10]$. These two T3SSs are involved in distinct pathogenic mechanisms during infection. The type III secretion system 1 (T3SS1) is required for cytotoxicity, whereas the type III secretion system 2 (T3SS2) is often responsible for enterotoxicity and intestinal fluid accumulation. The overall mechanism of pathogenesis in $V$. parahaemolyticus remains unclear.

$V$. parahaemolyticus infections are associated with multiple serotypes. Analysis of an outbreak in Calcutta in 1996 has identified a unique serotype clone, O3:K6, which is not previously isolated in that area [11]. After this report, O3:K6 isolates and its serovariants, defined to have identical genotype and molecular characteristics to those isolated in Calcutta, have been reported from foodborne outbreaks across the world. These serotypes were thought to be clonal derivatives of the O3:K6 serotype [12]. Recent multilocus sequence typing (MLST) data have further confirmed that multiple serotypes occur in a single genetic lineage [13].

Comparative genomics analysis of merely clinical strains of $V$. parahaemolyticus was described before [14]. However, the phylogenetic diversity and niche adaptation of $V$. parahaemolyticus at the species level is currently unknown. Strains isolated from environmental reservoirs could be as virulent as clinical strains in animal models [15], and the existing regulatory mechanisms of environmental $V$. parahaemolyticus could favor regulations of foreign virulence genes [16]. Nonetheless, the virulence potential of $V$. parahaemolyticus at the genome-wide level has not been described before. We sequenced the genomes of four O3:K6 and its serovariant clinical strains and five environmental strains of $V$. parahaemolyticus in Hong Kong. Genomes of a few environmental $V$. parahaemolyticus isolates have been announced recently [17-20], however, comparative genomics study has not been reported until now. Phylogenomic analysis of our local strains and other available genomes revealed a clear distinction between clinical and environmental strains.

\section{Results and discussion}

\section{Summary of genome sequencing data}

As summarized in Table 1, we have completed 454 genome sequencing of nine $V$. parahaemolyticus isolates

Table 1 List of $V$. parahaemolyticus clinical and environmental isolates analyzed in this study

\begin{tabular}{|c|c|c|c|c|c|c|c|}
\hline Strain & Specimen collection date & Source & tdh & trh & Serotype & CDS & Reference \\
\hline VIP4-0395 & $20 / 11 / 07$ & Local-Stool & + & - & O3:K6 & 4711 & This study \\
\hline VIP4-0439 & 10/09/08 & Local-Stool & + & - & O3:K6 & 4733 & This study \\
\hline VIP4-0445 & $26 / 09 / 08$ & Local-Stool & + & - & O3:K6 & 6226 & This study \\
\hline VIP4-0407 & $18 / 01 / 08$ & Local-Stool & + & - & O3:K59 & 4692 & This study \\
\hline AQ3810 & 1983 & Singapore & + & - & O3:K6 & 5458 & [11] \\
\hline AQ4037 & 1985 & Maldives & - & + & O3:K6 & 4447 & [9] \\
\hline RIMD2210633 & 1996 & Thailand & + & - & O3:K6 & 4831 & {$[12]$} \\
\hline Peru-466 & 1996 & Peru & + & - & O3:K6 & 4603 & [9] \\
\hline AN-5034 & 1998 & Banglandesh & + & - & O4:K68 & 4770 & [9] \\
\hline K5030 & 2005 & India & + & - & O3:K6 & 4606 & [9] \\
\hline VIP4-0430 & 03/07/08 & Local-Oyster & - & - & O4:K34 & 5698 & This study \\
\hline VIP4-0443 & $23 / 09 / 08$ & Local-Big eye fish & - & - & O2:UT & 5994 & This study \\
\hline VIP4-0219 & $13 / 04 / 06$ & Local-Salmon Sashimi & - & - & 01:UT & 4761 & This study \\
\hline VIP4-0444 & 23/09/08 & Local-Big eye fish & - & - & O11:UT & 4976 & This study \\
\hline VIP4-0447 & $22 / 10 / 08$ & Local-Oyster & - & - & O6:UT & 5091 & This study \\
\hline
\end{tabular}

UT: Untypeable. 
obtained in Hong Kong, including four clinical and five environmental strains. The average sequencing coverage was between $44 \times$ to $66 \times$. Genome sequences of these local $V$. parahaemolyticus strains were de novo assembled using Newbler 2.7 (Roche Diagnostics). Genome assembly of $V$. parahaemolyticus yielded 130 to 1205 contigs of N50 contig length ranging from $7 \mathrm{~kb}$ to 305 $\mathrm{kb}$. The clinical strains we sequenced were new O3:K6 and its serovariant (O3:K59), and they were positive for the $t d h$ gene but negative for the $t r h$ gene. Our environmental strains were all $t d h$ and trh negative. We have also included in our analyses six publicly available $V$. parahaemolyticus genomes, which are all clinical strains (Table 1) and two of them (AQ3810, AQ4037) pre-pandemic strains [14].

\section{Phylogenetic relationships}

Phylogenetic relationships among our $V$. parahaemolyticus isolates and the reference strains (Table 1) were examined using a genome-wide approach based on 169,998 single nucleotide polymorphisms (SNPs). Eighty nine percent of these SNPs were in coding regions, of which $25.8 \%$ were missense, $0.6 \%$ was nonsense, and $73.6 \%$ were synonymous. Genome-wide comparison readily resolved the relationship among the clinical and environmental isolates (Figure 1). Clinical strains were found to be more related compared to the environmental strains. Moreover, our local clinical isolates were highly similar to RIMD 2210633 isolated from Thailand in 1996, consistent with previous results using various molecular methods [21]

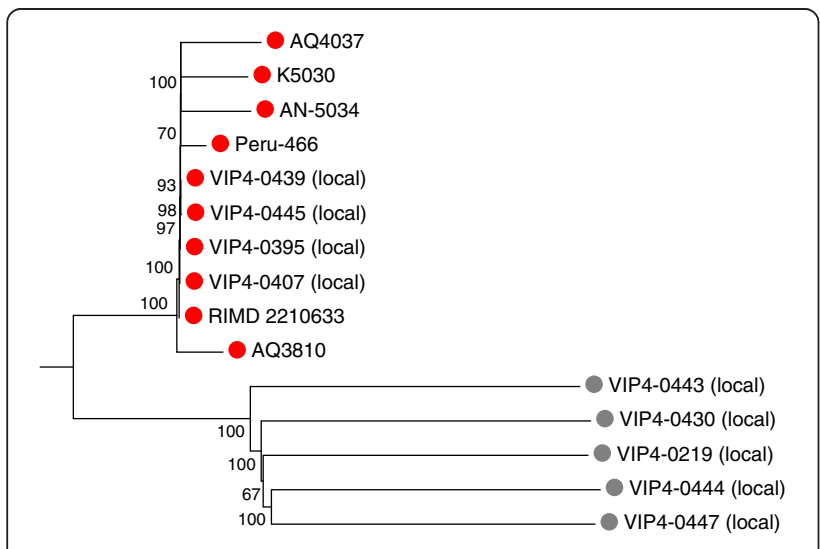

Figure 1 Neighbor-joining phylogenetic tree showing relationships among $V$. parahaemolyticus isolates inferred using SNP sites. Nodal supports were calculated from 500 bootstrap pseudoreplicates. The tree was rooted using Vibrio harveyi ATCC BAA-1116. The scale bar represents 0.002 substitutions per nucleotide position. Red color indicates clinical strains, and grey color indicates environmental strains.
[22] and further confirm clonality of new O3:K6 and its serovariants.

\section{Pan-genome analysis}

Comparative analysis of nine newly sequenced genomes and six publicly available genomes of $V$. parahaemolyticus could help us determine the global gene repertoire of the species. This can be described by its 'pan-genome' that includes a core genome containing genes present in all strains and a dispensable genome composed of genes absent from one or more strains and genes that are unique to some strains. Here, the core and pan-genomes of $V$. parahaemolyticus were identified using OrthoMCL [23].

Our result shows that the number of pan-genome gene families increased with the number of genomes analysed, indicating that $V$. parahaemolyticus harbors an open pan-genome (Figure 2). We have also analyzed the trend of new gene families, and we found that new genes will continue to be found with increasing of much more genomes.

\section{Gene-content analysis of $V$. parahaemolyticus}

By investigating the presence and absence of genes in $V$. parahaemolyticus, we found that most regions within their genomes were conserved (Figure 3). We then further analyzed 24 genomic regions that are unique to V. parahaemolyticus RIMD 2210633 [24], which mainly include two T3SS, seven pathogenic islands, and f237 prophages. Comparative genomics revealed that these 24 regions accounted for most of the varying regions. Five pathogenic islands (VPal-1, VPal-3, VPal-4, VPal-5, and VPal-6) were found absent in not only the environmental strains, but also two pre-pandemic clinical strains. VPal-7 was found absent in AQ4037 and other environmental strains.

Previously, microarray-based approach failed to detect genes responsible for pathogenesis of $V$. parahaemolyticus [25]. Traditional features also failed to differentiate clinical strains from environmental strains [26,27]. Here, analysis of these varying regions revealed the presence of only 11 regions in the clinical group, which were absent in the environmental group (Table 2). As expected, genes in T3SS2 were not found in all of the clinical strains, which further confirm that it is not reliable to simply use $t d h$, trh or genes in T3SS as virulence features [25]. However, a region located in chromosome II (984909-999901) contained genes from VPA0950 to VPA0957, which encode putative biofilm associated proteins and outer membrane proteins. A few genes including two type IV pili were previously described in biofilm formation of $V$. parahaemolyticus, however details of the process are still unexplored in this bacterium $[28,29]$. In other Vibrio species such as $V$. fischeri, biofilm formation was found to play an important role in 

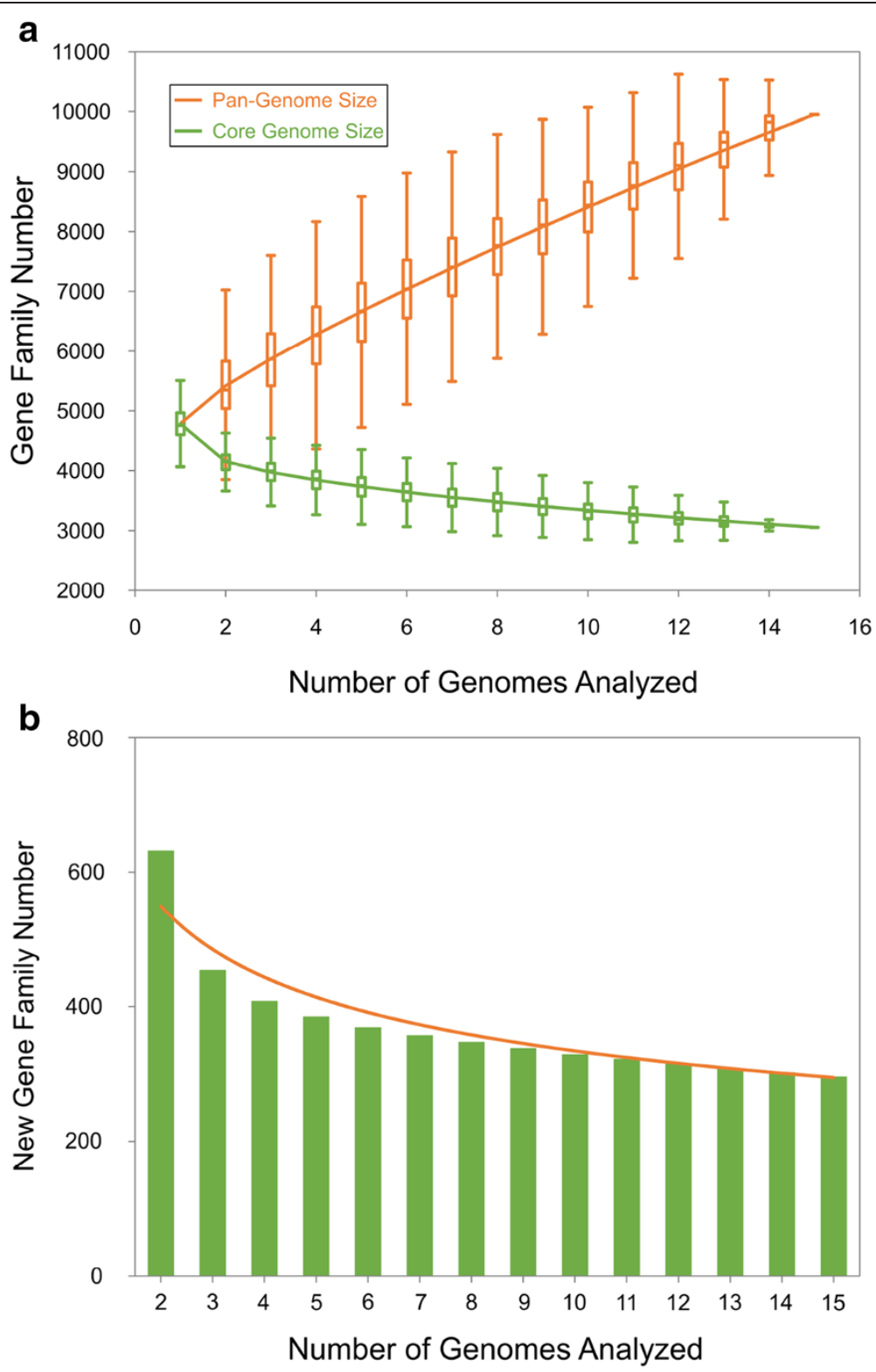

Figure 2 Gene repertoire analysis of $\boldsymbol{V}$. parahaemolyticus. (A) Pan-genome and core genome size accumulation. (B) New gene family accumulation. The green bars indicate the number of expected new gene family detected for a particular number of genomes analysed, and the orange line indicates the trend of expected new gene family with an increasing number of genomes.

host colonization [30]. It is suggested that gene clusters found in clinical strains of $V$. parahaemolyticus could also provide this bacterium with advantages in host adaptation. These gene clusters have not been found previously, and we suggest that they could act as new candidate virulence markers.

\section{A novel genomic island commonly found in clinical strains}

O3:K59 is a new serovariant of serotype O3:K6. It is recently reported that it could replace local serovariants and has caused pandemics in Chile and Peru [31,32].
Here we analyzed the genome of our O3:K59 strain VIP4-0407 and found a novel genomic island that is absent in $V$. parahaemolyticus RIMD 2210633 [33]. This genomic island was found in most of our clinical strains including AQ4037, K5030, AN-5034, Peru-466, VIP40439, VIP4-0395, VIP4-0407 and AQ3810, but was absent in our environmental strains. We defined this genomic island as Vibrio parahaemolyticus island-8 (VPal-8). It contained six CDSs (Table 3), one of which showing high similarity with an AraC family transcriptional regulator that caused virulence in a mouse infection model in $\mathrm{Myco-}$ bacterium tuberculosis [34]. ArcC family transcriptional 


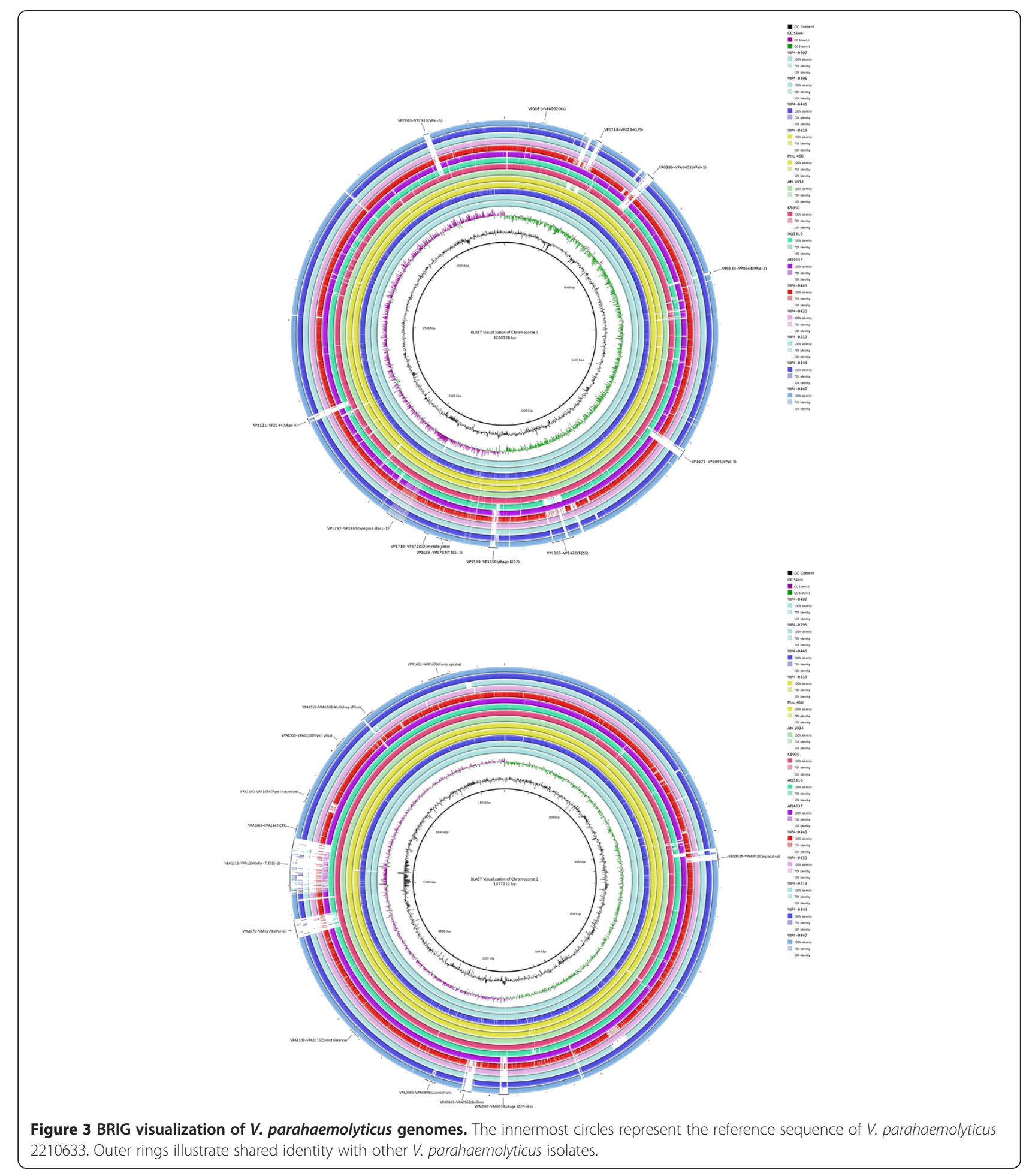

regulators could regulate T3SS genes to modulate bacterial virulence [35], We analyzed sequences in this genomic island to determine whether there is any T3SS secreted protein. Using a combination of available T3SS prediction tools including T3_MM [36], Effective T4 [37], and T3SS effector prediction [38], we found that the third protein in this genomic island showed positive results in all of the prediction tools.

We further investigated whether VPal-8 is widespread across the population of clinical strains. Using this genomic island as a probe to perform southern hybridization on an additional population of 40 clinical 
Table 2 Genomic regions that found in clinical group only

\begin{tabular}{lcccccl}
\hline Reference No. & Chr & Start & End & Length & Features in this regions* & Descriptions \\
\hline NC_004603.1 & Chr1 & 1630527 & 1631678 & 1151 & VP1517, VP1518 & Rhs-family protein \\
NC_004603.1 & Chr1 & 1632125 & 1632898 & 773 & VP1520, VP1521 & Hypothetical protein \\
NC_004605.1 & Chr2 & 433304 & 434859 & 1555 & VPA0442,VPA0443 & Hypothetical protein \\
NC_004605.1 & Chr2 & 436019 & 437350 & 1331 & VPA0445, VPA0446 & Methylamine utilization protein MauG precursor \\
NC_004605.1 & Chr2 & 438755 & 439983 & 1228 & VPA0447 & Hypothetical protein \\
NC_004605.1 & Chr2 & 823596 & 824400 & 804 & VPA0796 & L-allo-threonine aldolase \\
NC_004605.1 & Chr2 & 984909 & 986361 & 1452 & VPA0950, VPA0951 & Hypothetical protein \\
NC_004605.1 & Chr2 & 987912 & 990219 & 2307 & VPA0952, VPA0953(partial) & Biofilm-associated surface protein \\
NC_004605.1 & Chr2 & 994472 & 995346 & 874 & VPA0953 (partial) & Biofilm-associated surface protein \\
NC_004605.1 & Chr2 & 995407 & 998918 & 3511 & VPA0954, VPA0955, VPA0956, VPA0957 (partial) & Agglutination protein,outer membrane protein \\
NC_004605.1 & Chr2 & 998994 & 999901 & 907 & VPA0957 (partial) & Transporter binding protein \\
\hline
\end{tabular}

*The gene name is based on annotation of Vibrio parahaemolyticus RIMD 2210633.

strains, we found that this novel genomic island can be found in 29 of the clinical strains (Additional file 1: Table S1). Therefore, we suggest that the genomic island found in this study may be related to the virulence of $V$. parahaemolyticus.

\section{sRNAs and CRISPR element analysis}

CRISPRs (Clustered regularly interspaced short palindromic repeats) could play important roles in the interaction of bacteria and mobile genetic elements [39]. We annotated CRISPR elements in $V$. parahaemolyticus using CRISPR finder [40] and found a total of six CRISPR elements (Table 4). Our environmental strains were found to harbor fewer CRISPR types, whereas at least two CRISPRs were found in each of the clinical strains.

sRNAs are a class of non-coding RNAs in bacteria and are important post-transcriptional regulators in multiple crucial biological processes such as biofilm formation, quorum sensing and virulence [41,42]. We determined the sRNA sequences in our $V$. parahaemolyticus strains and found that most sRNAs were highly conserved (Additional file 2: Table S2). However, a few sRNAs such as GcvB and STnc1460 were only present in a few strains, indicating that these sRNAs may mediate some strain-specific regulations.

\section{Biphenyl degradation pathway identified in} environmental strains

We annotated the subsystems of $V$. parahaemolyticus genomes using the RAST Server [43] (Figure 4). Five subsystems were found enriched in the environmental strains, including Metabolism of Aromatic Compounds $(p=1.12 E 06)$, Protein metabolism $(p=0.01)$, Iron acquisition and metabolism $(\mathrm{p}=0.027)$, Phages and Prophages $(\mathrm{p}=0.041)$ and Sulfur Metabolism $(\mathrm{p}=0.046)$. The most enriched subsystem "Metabolism of Aromatic Compounds" contained an average of six genes in clinical strains, but an average of 10 genes in the environmental strains.

We further examined this category to investigate which pathway has contributed most of the differences. Genes involved in biphenyl degradation pathway (BphE1, BphC, Bphj2) were found in all of our environmental strains but not in the clinical strains, except the prepandemic strain AQ3810 (Table 5). The presence of the unique biphenyl degradation pathway in our environmental strains is possibly caused by the widespread of polychlorinated biphenyls in the environment [44].

\section{Virulence potential of environmental strains}

$V$. parahaemolyticus harbors many virulence factors including TDH, TRH and two T3SSs, which are generally pathogenic. Here we report the distribution of virulence

Table 3 Gene clusters in small genomic island found in V. parahaemolyticus

\begin{tabular}{ccccccc}
\hline No. & Accession ID & Start & End & No. of aa* & Homolog accession no. & Description \\
\hline 1 & AXNM01000034 & 96777 & 97262 & 162 & NP_797023.1 & SsrA-binding protein \\
2 & AXNM01000034 & 97884 & 99086 & 401 & ZP_05776346.1 & Phage integrase family protein \\
3 & AXNM01000034 & 99645 & 100427 & 261 & ZP_05888734.2 & Putative cyclic diguanylate phosphodiesterase (EAL) domain protein \\
4 & AXNM01000034 & 100420 & 101163 & 248 & WP_025628220.1 & AraC family transcriptional regulator \\
5 & AXNM01000034 & 102145 & 101300 & 282 & ZP_01990379.1 & Hypothetical protein \\
6 & AXNM01000034 & 103426 & 104361 & 312 & ZP_01990406.1 & Hypothetical protein \\
\hline
\end{tabular}

*Amino acids. 
Table 4 Distribution of CRISPRs in V. parahaemolyticus strains

\begin{tabular}{|c|c|c|c|c|c|c|c|}
\hline Strain & Source & CRISPR 1 & CRISPR 2 & CRISPR 3 & CRISPR 4 & CRISPR 5 & CRISPR 6 \\
\hline RIMD 2210633 & Clinical & + & - & + & - & - & - \\
\hline AQ3810 & Clinical & - & + & + & - & - & + \\
\hline Peru 466 & Clinical & + & + & - & - & - & - \\
\hline K5030 & Clinical & + & - & + & - & - & - \\
\hline AQ4037 & Clinical & - & - & + & - & + & - \\
\hline AN-5034 & Clinical & + & - & + & - & - & - \\
\hline VIP4-0439 & Clinical & + & - & + & + & - & - \\
\hline VIP4-0395 & Clinical & + & - & + & - & - & - \\
\hline VIP4-0407 & Clinical & + & + & - & - & - & - \\
\hline VIP4-0445 & Clinical & + & + & - & - & - & - \\
\hline VIP4-0444 & Environmental & - & + & - & - & + & - \\
\hline VIP4-0219 & Environmental & - & - & + & - & - & - \\
\hline VIP4-0447 & Environmental & - & - & - & - & - & - \\
\hline VIP4-0430 & Environmental & - & - & - & - & - & - \\
\hline VIP4-0443 & Environmental & - & - & - & - & - & - \\
\hline
\end{tabular}

factors and prophages in the genomes of our $V$. parahaemolyticus strains to reveal their pathogenic potential.

We predicted prophage regions in $V$. parahaemolyticus using the PHAST webserver [45] and found that clinical strains were restricted to a few prophage elements. The pandemic group of clinical strains possessed a filamentous prophage $\mathrm{f} 237$, which could increase virulence by adhering to intestinal cells [46]. A similar prophage was also found in the AQ3047 strain. In contrast, many diverse prophage elements were found in our environmental strains
(Table 6). Six new prophages were found in four of our environmental strains, which harbor genes that could enhance the fitness for survival in specific environments. We further examined the relationship between the number of prophages and CRISPR in our strains. We analyzed the spacer sequences of CRISPRs and eight unique spacers sequence were found. However, we could not find any homologous prophage regions that could match with these spacer sequences in the respective strains. This could be explained by the possibility that the CRISPRs of Vibrio

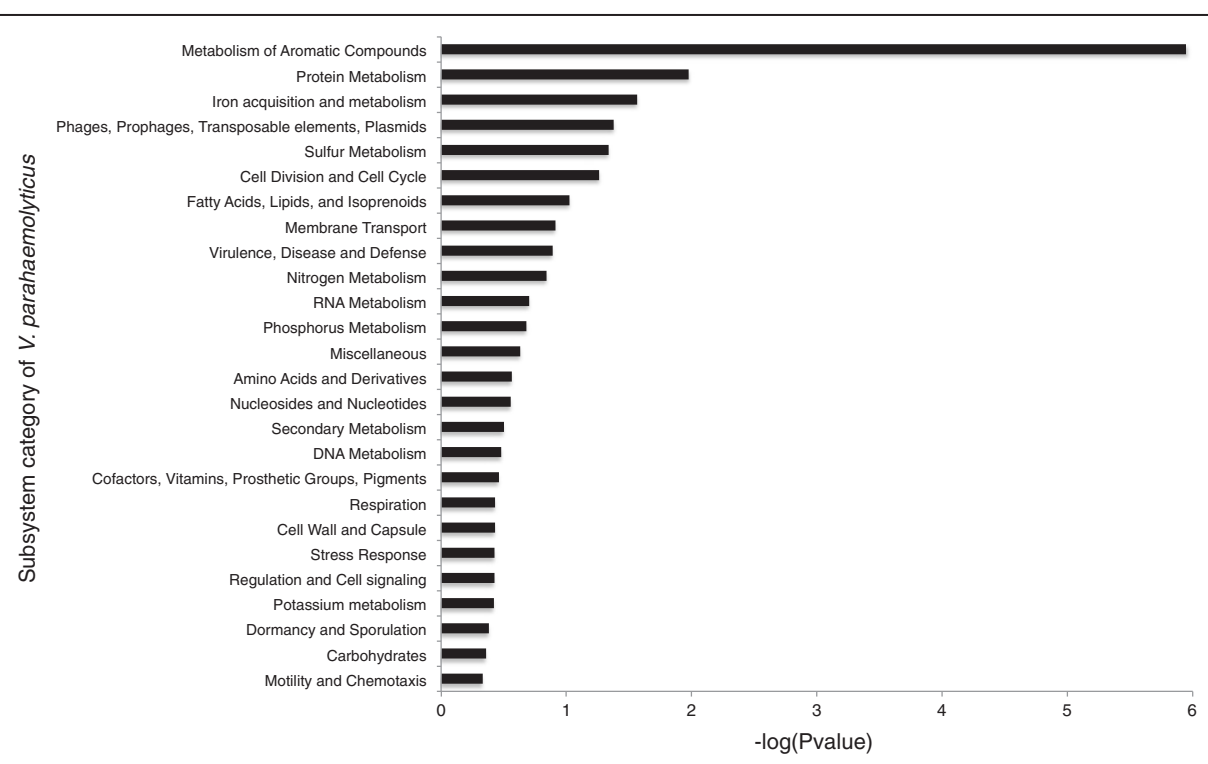

Figure 4 Subsystem annotation of $\boldsymbol{V}$. parahaemolyticus. The $y$-axis represents subsystems annotated in the RAST server whereas the $x$-axis shows the respective - $\log (P)$ values. P-values were calculated to examine if there is significant difference in the numbers between the subsystems of environmental strains and clinical strains. The subsystem "metabolism of aromatic compounds" was shown to have a most significant P-value (P-value $<0.05,-\log P>1.3)$. 
Table 5 Detection of biphenyl degradation pathway

\begin{tabular}{lcccc} 
genes & & & & \\
\hline Strains* & Source & BphE1 & BphC & Bphj2 \\
\hline VIP4-0444 & Environmental & + & + & + \\
VIP4-0219 & Environmental & + & + & + \\
VIP4-0430 & Environmental & + & + & + \\
VIP4-0443 & Environmental & + & + & + \\
VIP4-0447 & Environmental & + & + & + \\
AQ3810 & Clinical & + & + & + \\
\hline
\end{tabular}

*Only strains with positive results are shown here.

parahaemolyticus could inhibit the prophage insertion to the host genome. Indeed, an inverse correlation of CRISPRs with the number of prophage was also described in Streptococcus pyogenes [47].

toxR gene, located next to toxS gene, involves in the regulation of many virulence-associated genes of $V$. parahaemolyticus [48]. Variation in the toxR sequence can be used to differentiate phylogenetically distinct clusters in $V$. parahaemolyticus. Seven base positions were previously identified to distinguish the O3:K6 isolates before 1995 from the new O3:K6 clones within a 1346-bp region [49]. We further investigated the variations between these base positions within our $V$. parahaemolyticus isolates. In all of the seven new O3:K6 clones, the bases were perfectly mapped to the reference ones (Table 7). However, another environmental strain (VIP4-0443) also had the same sequence bases with the new O3:K6 clones, suggesting that it may also harbor virulence potential. We then further examined whether there are any other virulence proteins in this strain. A total of 264 candidate virulence genes were found (Additional file 3: Table S3), which further confirm that this environmental strain could have virulence potential.

\section{Construction of a genome sequence database for V. parahaemolyticus}

A $V$. parahaemolyticus genome database was constructed here based on the Ensembl genome annotation system using Perl scripts (Figure 5). Genome DNA sequences can be input into the platform via a user-friendly web-based interface. Our web-based database allows comparative analysis and data mining using available $V$. parahaemolyticus genome data. A suite of useful computational tools is now available for data analysis such as MLST and detection of genetic variations. The web-based database is now launched as a publicly accessible domain (http://kwanlab. bio.cuhk.edu.hk/vp).

\section{Conclusions}

This is the first study on the genome dynamics of $V$. parahaemolyticus at the species level. We have three main conclusions. First, by comparing the genome sequences, we found that our clinical strains were phylogenetically distinct from the environmental strains. We discovered a new gene cluster belonging to the biofilm associated proteins of $V$. parahaemolyticus. We suggest that this novel gene cluster is a new virulence marker differentiating clinical from environmental strains. We also found a novel genomic island (VPal-8) that frequently distributed in the clincial strains. Second, by analyzing the virulence features and prophage elements of our environmental strains on a genome-wide scale, we discovered a new type of toxRS in one of the environmental strains and a diverse array of prophage elements in the environmental strains. These results indicate that the environmental strains studied could have virulence potential. Third, we set up an Ensemblbased genome database for $V$. parahaemolyticus to provide a unified and user-friendly platform to access all currently available genome sequences and annotations of this bacterium.

\section{Methods}

\section{Ethics statement and isolate collection}

Nine isolates of $V$. parahaemolyticus were selected from the bacterial archive maintained at the microbiology laboratory of the Department of Health of Hong Kong and were characterized for genome sequencing. The study was approved by the Joint CUHK-NTEC Clinical Research Ethical Committee at the Prince of Wales Hospital in Hong Kong. Written informed consent was obtained from all the studied subjects for sample collection and subsequent analysis.

\section{DNA sequencing}

Whole-genome shotgun sequencing was performed using a 454 Genome Sequencer (GS) FLX-Titanium (Roche Diagnostics, US). DNA of each selected isolate was extracted from culture and subjected to sequencing library preparation according to the manufacturer's recommended protocols. Libraries of processed genomic DNA fragments immobilized on DNA capture beads were individually sequenced on a PicoTiterPlate device. Bases sequenced and the corresponding quality values were called and delivered in a standard format by GS-FLX-Titanium system for downstream bioinformatic analyses. The remaining genome gaps were filled by the GS-FLXprovided paired-end approach and/or primer walking. The primer walking approach involved design of primers flanking the gaps and PCR to amplify the DNA fragments covering the gaps. PCR products were then directly sequenced using the ABI DNA Sequencer (Applied Biosystems, US).

\section{Sequence assembly and annotation}

Raw sequence reads were first filtered to remove lowquality reads. Sequence assembly was performed on the 
Table 6 Distribution of prophages in V. parahaemolyticus strains

\begin{tabular}{|c|c|c|c|c|c|c|c|c|c|}
\hline Strains* & Source & Prophage 1 (f237) & Prophage 2 (f237-like) & Prophage 3 & Prophage 4 & Prophage 5 & Prophage 6 & Prophage 7 & Prophage 8 \\
\hline RIMD 2210633 & Clinical & + & & & & & & & \\
\hline Peru 466 & Clinical & + & & & & & & & \\
\hline K5030 & Clinical & + & & & & & & & \\
\hline AN-5034 & Clinical & + & & & & & & & \\
\hline VIP4-0439 & Clinical & + & & & & & & & \\
\hline VIP4-0395 & Clinical & + & & & & & & & \\
\hline VIP4-0407 & Clinical & + & & & & & & & \\
\hline VIP4-0445 & Clinical & + & & & & & & & \\
\hline AQ4037 & Clinical & - & + & & & & & & \\
\hline VIP4-0444 & Environmental & - & & 19312/19312 & $8265 / 8265$ & & & & \\
\hline VIP4-0447 & Environmental & - & & $12018 / 19312$ & $6500 / 8265$ & $91629 / 91629$ & & & \\
\hline VIP4-0219 & Environmental & - & & & & & $34088 / 34088$ & & \\
\hline VIP4-0430 & Environmental & - & & & & & & $58355 / 58355$ & $48569 / 48569$ \\
\hline
\end{tabular}

*VIP4-0443,AQ3810 was excluded because no prophage was found in this strain. Numbers denote the length of homolog sequences / the length of intact prophage. 
Table 7 Base variations in the toxRS sequence of selected $V$. parahaemolyticus strains

\begin{tabular}{|c|c|c|c|c|c|c|c|c|}
\hline \multirow[t]{2}{*}{ Strains } & \multirow[t]{2}{*}{ Sources } & \multicolumn{7}{|c|}{ Position at the toxRS sequences } \\
\hline & & 576 & 900 & 1002 & 1196 & 1214 & 1244 & 1463 \\
\hline RIMD 2210633 & Clinical & A & A & $\mathrm{T}$ & $\mathrm{T}$ & $\mathrm{T}$ & A & $\mathrm{T}$ \\
\hline Peru 466 & Clinical & A & A & $\mathrm{T}$ & $\mathrm{T}$ & $\mathrm{T}$ & A & $\mathrm{T}$ \\
\hline K5030 & Clinical & A & A & $\mathrm{T}$ & T & $\mathrm{T}$ & A & $\mathrm{T}$ \\
\hline AN-5034 & Clinical & A & A & $\mathrm{T}$ & $\mathrm{T}$ & $\mathrm{T}$ & A & $\mathrm{T}$ \\
\hline VIP4-0439 & Clinical & A & A & $\mathrm{T}$ & T & $\mathrm{T}$ & A & $\mathrm{T}$ \\
\hline VIP4-0395 & Clinical & A & A & $\mathrm{T}$ & $\mathrm{T}$ & $\mathrm{T}$ & A & $T$ \\
\hline VIP4-0407 & Clinical & A & A & $\mathrm{T}$ & $\mathrm{T}$ & $\mathrm{T}$ & A & $\mathrm{T}$ \\
\hline VIP4-0445 & Clinical & A & A & $\mathrm{T}$ & $\mathrm{T}$ & $\mathrm{T}$ & A & $T$ \\
\hline AQ4037 & Clinical & G & G & C & C & A & G & A \\
\hline AQ3810 & Clinical & G & G & C & C & A & G & A \\
\hline VIP4-0444 & Environmental & G & G & C & C & A & G & A \\
\hline VIP4-0447 & Environmental & G & G & C & C & A & G & A \\
\hline VIP4-0219 & Environmental & G & G & C & C & A & G & A \\
\hline VIP4-0430 & Environmental & G & G & C & C & A & G & A \\
\hline VIP4-0443 & Environmental & $A$ & A & $\mathrm{T}$ & $\mathrm{T}$ & $\mathrm{T}$ & A & $\mathrm{T}$ \\
\hline
\end{tabular}

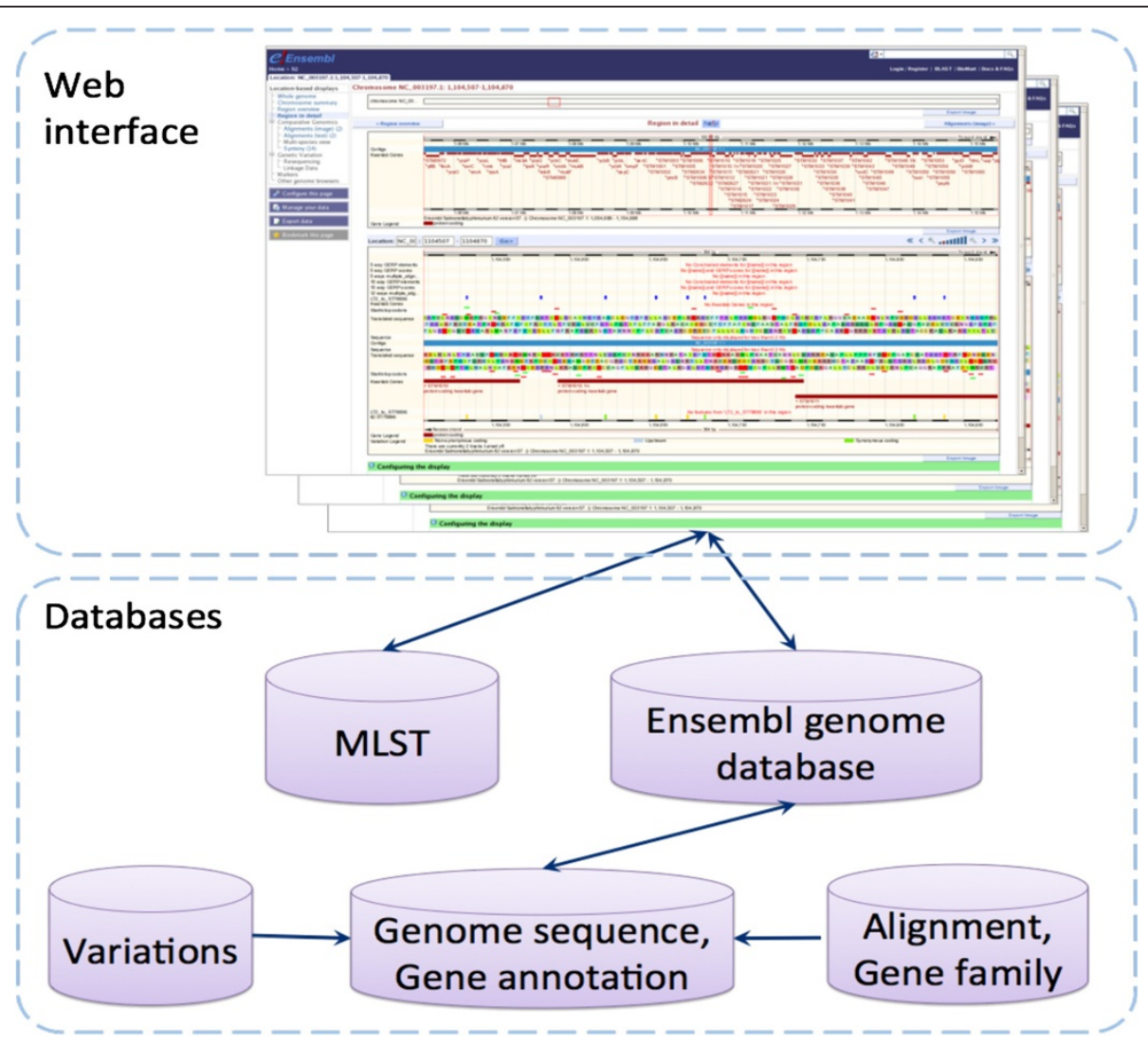

Figure 5 Architecture of the Ensembl-based genome database for V. parahaemolyticus. 
remaining clean reads de novo using the GS Newbler 2.7. The genome sequences were annotated using the RAST webserver. Contigs were reordered and genome comparisons were carried out using mauve program [50]. BRIG was used for genome alignment visualization [51]. Prophage elements of $V$. parahaemolyticus were predicted using PHAST [45].

\section{Phylogenetic tree construction}

SNPs among our isolates and several foreign strains were identified using GS Reference Mapper (Roche Diagnostics). SNPs detected among all strains were concatenated together and a neighbor-joining phylogenetic tree was constructed using MEGA 6 [52] with 500 bootstrap pseudoreplicates. Vibrio harveyi ATCC BAA-1116 was used as outgroup for defining the root.

\section{Small RNAs and CRISPRs}

A list of small RNA sequences was retrieved from the BSRD database [42]. sRNA homologs in our V. parahaemolyticus isolates were then identified using BLASTn. The e-value of BLAST was set to 1e-5. Annotation of CRISPR elements was done using CRISPRfinder [40].

\section{Experimental validation of genomic island}

Two sets of primers were designed for PCR-based detection of genomic island in a population of clinical $V$. parahaemolyticus. These clinical strains were previously used for microarray-based analysis [53].

\section{Availability of supporting data}

Data of this Whole Genome Shotgun project have been deposited at GenBank under the accessions AXNJ00000000, AXNK00000000, AXNL00000000, AXNM00000000, AXN O00000000, AXNP00000000, AXNQ00000000, AXNR00 000000, and AXNS00000000. The phylogenetic tree and associated data matrix are available in TreeBASE database (Accession URL: http://purl.org/phylo/treebase/phylows/ study/TB2:S16780).

\section{Additional files}

Additional file 1: Table S1. Primers used in PCR detection of genomic island in $V$. parahaemolyticus isolates.

Additional file 2: Table S2: Distribution of sRNA sequences in $\mathrm{V}$. parahaemolyticus strains.

Additional file 3: Table S3. Homologous virulence-associated genes in environmental strain VIP4-0443.

\section{Competing interests}

The authors declare that they have no competing interests.

\section{Authors' contributions}

Conceived and designed the project: HSK. Analyzed and interpreted the data: LL, HCW. Performed the genomic island experiments: HCW. Constructed the genome database: WN. Performed 454 sequencing: PTWL.
Provided the bacterial strains: KMK. Drafted the manuscript: LL. Revised the manuscript: LL, HSK, HCW, MKC. All authors have read and approved the final manuscript.

\section{Acknowledgements}

This work was supported by the Research Fund for the Control of Infectious Diseases (CHP-PH-06) from the Food and Health Bureau of the Hong Kong SAR, ROC

\section{Author details}

${ }^{1}$ School of Life Sciences, The Chinese University of Hong Kong, Shatin, New Territories, Hong Kong SAR, People's Republic of China. ${ }^{2}$ Department of Microbiology, Soochow University, Taipei 111, Taiwan. ${ }^{3}$ Stanley Ho Centre for Emerging Infectious Diseases, JC School of Public Health, Faculty of Medicine, The Chinese University of Hong Kong, Hong Kong SAR, People's Republic of China. ${ }^{4}$ Present address: Institute for Molecular Infection Biology, University of Würzburg, Würzburg, Germany.

Received: 5 September 2014 Accepted: 11 December 2014 Published: 18 December 2014

\section{References}

1. Scientific Committee on Enteric Infections and Foodborne Diseases. 2008. Foodborne illness - Intersection between clinical and public health approaches, Centre for Health Protection, Hong Kong SAR. http://www. chp.gov.hk/files/pdf/foodborne_illness-intersection_between_clinical_ and_public_health_approaches_r.pdf.

2. Nair GB, Ramamurthy T, Bhattacharya SK, Dutta B, Takeda Y, Sack DA: Global dissemination of Vibrio parahaemolyticus serotype 03:K6 and its serovariants. Clin Microbiol Rev 2007, 20:39-48.

3. Miyamoto $Y$, Nakamura K, Takizawa K: Seasonal distribution of oceanomonas spp., halophilic bacteria, in the coastal sea. its significance in epidemiology and marine industry. Jpn J Microbiol 1962, 6:141-158.

4. Miyamoto Y, Kato T, Obara Y, Akiyama S, Takizawa K, Yamai S: In vitro hemolytic characteristic of Vibrio parahaemolyticus: its close correlation with human pathogenicity. J Bacterio/ 1969, 100:1147-1149.

5. Baker-Austin C, Stockley L, Rangdale R, Martínez-Urtaza J: Environmental occurrence and clinical impact of Vibrio vulnificus and Vibrio parahaemolyticus: a European perspective. Environ Microbiol Rep 2010, 2:7-18.

6. Raimondi F, Kao JP, Fiorentini C, Fabbri A, Donelli G, Gasparini N, Rubino A, Fasano A: Enterotoxicity and cytotoxicity of Vibrio parahaemolyticus thermostable direct hemolysin in in vitro systems. Infect Immun 2000, 68:3180-3185.

7. Honda T, Ni YX, Miwatani T: Purification and characterization of a hemolysin produced by a clinical isolate of Kanagawa phenomenonnegative Vibrio parahaemolyticus and related to the thermostable direct hemolysin. Infect Immun 1988, 56:961-965.

8. Lynch T, Livingstone S, Buenaventura E, Lutter E, Fedwick J, Buret AG, Graham D, DeVinney R: Vibrio parahaemolyticus disruption of epithelial cell tight junctions occurs independently of toxin production. Infect Immun 2005, 73:1275-1283.

9. Piñeyro $P$, Zhou $X$, Orfe $L H$, Friel PJ, Lahmers $K$, Call DR: Development of two animal models to study the function of Vibrio parahaemolyticus type III secretion systems. Infect Immun 2010, 78:4551-4559.

10. Hiyoshi H, Kodama T, lida T, Honda T: Contribution of Vibrio parahaemolyticus virulence factors to cytotoxicity, enterotoxicity, and lethality in mice. Infect Immun 2010, 78:1772-1780.

11. Okuda J, Ishibashi M, Hayakawa E, Nishino T, Takeda Y, Mukhopadhyay AK, Garg S, Bhattacharya SK, Nair GB, Nishibuchi M: Emergence of a unique O3: $\mathrm{K} 6$ clone of Vibrio parahaemolyticus in Calcutta, India, and isolation of strains from the same clonal group from southeast asian travelers arriving in Japan. J Clin Microbiol 1997, 35:3150-3155.

12. Chowdhury NR, Chakraborty S, Ramamurthy T, Nishibuchi M, Yamasaki S, Takeda Y, Nair GB: Molecular evidence of clonal Vibrio parahaemolyticus pandemic strains. Emerg Infect Dis 2000, 6:631-636.

13. Chowdhury NR, Stine OC, Morris JG, Nair GB: Assessment of evolution of pandemic Vibrio parahaemolyticus by multilocus sequence typing. $J$ Clin Microbiol 2004, 42:1280-1282. 
14. Chen Y, Stine OC, Badger JH, Gil Al, Nair GB, Nishibuchi M, Fouts DE: Comparative genomic analysis of Vibrio parahaemolyticus: serotype conversion and virulence. BMC Genomics 2011, 12:294

15. Chatzidaki-Livanis M, Hubbard MA, Gordon K, Harwood VJ, Wright AC: Genetic distinctions among clinical and environmental strains of Vibrio vulnificus. Appl Environ Microbiol 2006, 72:6136-6141.

16. Mahoney JC, Gerding MJ, Jones SH, Whistler CA: Comparison of the pathogenic potentials of environmental and clinical Vibrio parahaemolyticus strains indicates a role for temperature regulation in virulence. Appl Environ Microbiol 2010, 76:7459-7465.

17. Liu M, Chen S: Draft genome sequence of Vibrio parahaemolyticus V110, isolated from shrimp in Hong Kong. Genome Announc 2013, 1:e00300-e00313.

18. Jun JW, Kim JH, Choresca CH, Shin SP, Han JE, Park SC: Draft genome sequence of Vibrio parahaemolyticus SNUVpS-1 isolated from Korean Seafood. Genome Announc 2013, 1. doi:10.1128/genomeA.00132-12. Epub 2013 Feb 7.

19. Kalburge SS, Polson SW, Boyd Crotty K, Katz L, Turnsek M, Tarr CL, MartinezUrtaza J, Boyd EF: Complete genome sequence of Vibrio parahaemolyticus environmental strain UCM-V493. Genome Announc 2014, 2. doi:10.1128/genomeA.00159-14.

20. Kumar BK, Deekshit VK, Rai P, Gurtler V, Karunasagar I, Karunasagar I: Draft genome sequence of trh + Vibrio parahaemolyticus VP-49, isolated from seafood harvested along the Mangalore Coast, India. Genome Announc 2014, 2:e00607-e00614.

21. Wong HC, Liu SH, Wang TK, Lee CL, Chiou CS, Liu DP, Nishibuchi M, Lee BK: Characteristics of Vibrio parahaemolyticus O3:K6 from Asia. Appl Environ Microbiol 2000, 66:3981-3986.

22. Matsumoto C, Okuda J, Ishibashi M, Iwanaga M, Garg P, Rammamurthy T, Wong HC, DePaola A, Kim YB, Albert MJ, Nishibuchi M: Pandemic spread of an O3:K6 clone of Vibrio parahaemolyticus and emergence of related strains evidenced by arbitrarily primed PCR and toxRS sequence analyses. J Clin Microbiol 2000, 38:578-585.

23. Li L: OrthoMCL: identification of Ortholog groups for Eukaryotic Genomes. Genome Res 2003, 13:2178-2189.

24. Boyd EF, Cohen A, Naughton LM, Ussery DW, Binnewies TT, Stine OC, Parent MA: Molecular analysis of the emergence of pandemic Vibrio parahaemolyticus. BMC Microbio/ 2008, 8:110.

25. Izutsu K, Kurokawa K, Tashiro K, Kuhara S, Hayashi T, Honda T, lida T: Comparative genomic analysis using microarray demonstrates a strong correlation between the presence of the 80-kilobase pathogenicity island and pathogenicity in Kanagawa phenomenon-positive Vibrio parahaemolyticus strains. Infect Immun 2008, 76:1016-1023.

26. Tsai S-E, Jong K-J, Tey YH, Yu W-T, Chiou C-S, Lee Y-S, Wong H-C: Molecular characterization of clinical and environmental Vibrio parahaemolyticus isolates in Taiwan. Int J Food Microbiol 2013, 165:18-26.

27. Yu W-T, Jong K-J, Lin Y-R, Tsai S-E, Tey $Y H$, Wong H-C: Prevalence of Vibrio parahaemolyticus in oyster and clam culturing environments in Taiwan. Int J Food Microbiol 2013, 160:185-192.

28. Enos-Berlage JL, Guvener ZT, Keenan CE, McCarter LL: Genetic determinants of biofilm development of opaque and translucent Vibrio parahaemolyticus. Mol Microbiol 2005, 55:1160-1182.

29. Shime-Hattori A, lida T, Arita M, Park K-S, Kodama T, Honda T: Two type IV pili of Vibrio parahaemolyticus play different roles in biofilm formation. FEMS Microbiol Lett 2006, 264:89-97.

30. Yildiz FH, Visick KL: Vibrio biofilms: so much the same yet so different. Trends Microbiol 2009, 17:109-118.

31. Velazquez-Roman J, León-Sicairos N, de Jesus Hernández-Díaz L, CanizalezRoman A: Pandemic Vibrio parahaemolyticus O3:K6 on the American continent. Front Cell Infect Microbiol 2013, 3:110.

32. García K, Torres R, Uribe P, Hernández C, Rioseco ML, Romero J, Espejo RT: Dynamics of clinical and environmental Vibrio parahaemolyticus strains during seafood-related summer diarrhea outbreaks in southern Chile. Appl Environ Microbiol 2009, 75:7482-7487.

33. Makino K, Oshima K, Kurokawa K, Yokoyama K, Uda T, Tagomori K, lijima Y, Najima M, Nakano M, Yamashita A, Kubota Y, Kimura S, Yasunaga T, Honda $T$, Shinagawa $H$, Hattori M, lida T: Genome sequence of Vibrio parahaemolyticus: a pathogenic mechanism distinct from that of $\mathrm{V}$ cholerae. Lancet 2003, 361:743-749.
34. Frota CC, Papavinasasundaram KG, Davis EO, Colston MJ: The AraC family transcriptional regulator Rv1931c plays a role in the virulence of Mycobacterium tuberculosis. Infect Immun 2004, 72:5483-5486.

35. Plano GV: Modulation of AraC family member activity by protein ligands. Mol Microbiol 2004, 54:287-290.

36. Wang $Y$, Sun M, Bao H, White AP: T3_MM: a Markov model effectively classifies bacterial type III secretion signals. PLOS One 2013, 8:e58173.

37. Arnold R, Brandmaier S, Kleine F, Tischler P, Heinz E, Behrens S, Niinikoski A Mewes H-W, Horn M, Rattei T: Sequence-based prediction of type III secreted proteins. PLoS Pathog 2009, 5:e1000376.

38. Löwer M, Schneider G: Prediction of type III secretion signals in genomes of gram-negative bacteria. PLoS One 2009, 4:e5917.

39. Westra ER, Swarts DC, Staals RHJ, Jore MM, Brouns SJJ, van der Oost J: The CRISPRs, they are a-changin': how prokaryotes generate adaptive immunity. Annu Rev Genet 2012, 46:311-339.

40. Grissa I, Vergnaud G, Pourcel C: CRISPRFinder: a web tool to identify clustered regularly interspaced short palindromic repeats. Nucleic Acids Res 2007, 35:W52-W57.

41. Storz G, Vogel J, Wassarman KM: Regulation by small RNAs in bacteria: expanding frontiers. Mol Cell 2011, 43:880-891.

42. Li L, Huang D, Cheung MK, Nong W, Huang Q, Kwan H-S: BSRD: a repository for bacterial small regulatory RNA. Nucleic Acids Res 2013, 41:D233-D238.

43. Overbeek R, Olson R, Pusch GD, Olsen GJ, Davis JJ, Disz T, Edwards RA, Gerdes S, Parrello B, Shukla M, Vonstein V, Wattam AR, Xia F, Stevens R: The SEED and the Rapid Annotation of microbial genomes using Subsystems Technology (RAST). Nucleic Acids Res 2014, 42:D206-D214.

44. Abramowicz DA: Aerobic and anaerobic PCB biodegradation in the environment. Environ Health Perspect 1995, 103:97-99.

45. Zhou Y, Liang Y, Lynch KH, Dennis JJ, Wishart DS: PHAST: a fast phage search tool. Nucleic Acids Res 2011, 39:W347-W352.

46. Nasu H, lida T, Sugahara T, Yamaichi Y, Park KS, Yokoyama K, Makino K, Shinagawa $\mathrm{H}$, Honda T: A filamentous phage associated with recent pandemic Vibrio parahaemolyticus O3:K6 strains. J Clin Microbio/ 2000, 38:2156-2161

47. Nozawa T, Furukawa N, Aikawa C, Watanabe T, Haobam B, Kurokawa K, Maruyama F, Nakagawa I: CRISPR inhibition of prophage acquisition in Streptococcus pyogenes. PLoS One 2011, 6:e19543.

48. Whitaker WB, Parent MA, Boyd A, Richards GP, Boyd EF: The Vibrio parahaemolyticus ToxRS regulator is required for stress tolerance and colonization in a novel orogastric streptomycin-induced adult murine model. Infect Immun 2012, 80:1834-1845.

49. Okura M, Osawa R, Iguchi A, Arakawa E, Terajima J: Genotypic analyses of Vibrio parahaemolyticus and development of a pandemic group-specific multiplex PCR assay. J Clin Microbiol 2003, 41:4676-4682.

50. Darling AE, Mau B, Perna NT: progressiveMauve: multiple genome alignment with gene gain, loss and rearrangement. PLoS One 2010, 5:e11147.

51. Alikhan N-F, Petty NK, Ben Zakour NL, Beatson SA: BLAST Ring Image Generator (BRIG): simple prokaryote genome comparisons. BMC Genomics 2011, 12:402.

52. Tamura K, Stecher G, Peterson D, Filipski A, Kumar S: MEGA6: molecular evolutionary genetics analysis version 6.0. Mol Biol Evol 2013, 30:2725-2729.

53. Han H, Wong H-C, Kan B, Guo Z, Zeng X, Yin S, Liu X, Yang R, Zhou D: Genome plasticity of Vibrio parahaemolyticus: microevolution of the "pandemic group.". BMC Genomics 2008, 9:570.

doi:10.1186/1471-2164-15-1135

Cite this article as: Li et al:: Comparative genomic analysis of clinical and environmental strains provides insight into the pathogenicity and evolution of Vibrio parahaemolyticus. BMC Genomics 2014 15:1135. 